\title{
The effect of oat $\beta$-glucan on LDL-cholesterol, non-HDL-cholesterol and apoB for CVD risk reduction: a systematic review and meta-analysis of randomised-controlled trials
}

\author{
Hoang V. T. Ho ${ }^{1,5}$, John L. Sievenpiper ${ }^{1,2,3,4,5}$, Andreea Zurbau ${ }^{1,5}$, Sonia Blanco Mejia ${ }^{1,4,5}$, \\ Elena Jovanovski ${ }^{1,5}$, Fei Au-Yeung ${ }^{1,5}$, Alexandra L. Jenkins ${ }^{1}$ and Vladimir Vuksan ${ }^{1,2,3,5 *}$ \\ ${ }^{1}$ Clinical Nutrition and Risk Factor Modification Centre, St. Michael's Hospital, 30 Bond Street, Toronto, ON, Canada, M5B 1 W8 \\ ${ }^{2}$ Li Ka Shing Knowledge Institute, St. Michael's Hospital, 30 Bond Street, Toronto, ON, Canada, M5B 1 W8 \\ ${ }^{3}$ Division of Endocrinology \& Medicine, St. Michael's Hospital, 30 Bond Street, Toronto, ON, Canada, M5B 1 W8 \\ ${ }^{4}$ Toronto $3 D$ Knowledge Synthesis and Clinical Trials Unit, St. Michael's Hospital, Toronto, ON, Canada, M5B 1 W8 \\ ${ }^{5}$ Department of Nutritional Sciences, Faculty of Medicine, University of Toronto, 27 King's Circle, Toronto, ON, Canada, M5S 1 A1 \\ (Submitted 24 February 2016 - Final revision received 7 June 2016 - Accepted 22 July 2016 - First published online 11 October 2016 )
}

\section{Abstract}

Oats are a rich source of $\beta$-glucan, a viscous, soluble fibre recognised for its cholesterol-lowering properties, and are associated with reduced risk of CVD. Our objective was to conduct a systematic review and meta-analysis of randomised-controlled trials (RCT) investigating the cholesterol-lowering potential of oat $\beta$-glucan on LDL-cholesterol, non-HDL-cholesterol and apoB for the risk reduction of CVD. MEDLINE, Embase, CINAHL and Cochrane CENTRAL were searched. We included RCT of $\geq 3$ weeks of follow-up, assessing the effect of diets enriched with oat $\beta$-glucan compared with controlled diets on LDL-cholesterol, non-HDL-cholesterol or apoB. Two independent reviewers extracted data and assessed study quality and risk of bias. Data were pooled using the generic inverse-variance method with random effects models and expressed as mean differences with $95 \%$ CI. Heterogeneity was assessed by the Cochran's $Q$ statistic and quantified by the $I^{2}$-statistic. In total, fifty-eight trials $(n$ 3974) were included. A median dose of $3.5 \mathrm{~g} / \mathrm{d}$ of oat $\beta$-glucan significantly lowered LDL-cholesterol $(-0 \cdot 19 ; 95 \%$ CI $-0 \cdot 23$, $-0 \cdot 14 \mathrm{mmol} / \mathrm{l}, P<0 \cdot 00001)$, non-HDL-cholesterol $(-0 \cdot 20 ; 95 \% \mathrm{CI}-0 \cdot 26,-0 \cdot 15 \mathrm{mmol} / 1, P<0 \cdot 00001)$ and apoB $(-0 \cdot 03 ; 95 \% \mathrm{CI}-0 \cdot 05,-0 \cdot 02 \mathrm{~g} / \mathrm{l}$, $P<0.0001)$ compared with control interventions. There was evidence for considerable unexplained heterogeneity in the analysis of LDL-cholesterol $\left(I^{2}=79 \%\right)$ and non-HDL-cholesterol $\left(I^{2}=99 \%\right)$. Pooled analyses showed that oat $\beta$-glucan has a lowering effect on LDL-cholesterol, non-HDL-cholesterol and apoB. Inclusion of oat-containing foods may be a strategy for achieving targets in CVD reduction.

Key words: Oats: $\beta$-Glucan: Cholesterol-lowering properties: CVD: Systematic reviews and meta-analyses

Oats are a rich source of $\beta$-glucan, a viscous, soluble fibre recognised for its cholesterol-lowering properties. The attenuation of blood cholesterol levels by oats was first reported in 1963 in a study that substituted white bread for oat bread containing $140 \mathrm{~g}$ of rolled oats ${ }^{(1)}$. Since then, a large number of studies have been conducted to assess the effects of oats on cholesterol levels, especially LDL-cholesterol, for the reduction of CVD risk. On the basis of the extensive evidence relating an inverse association between $\beta$-glucan intake and LDLcholesterol, several countries have currently approved health claims of oat $\beta$-glucan and its LDL-cholesterol-lowering effect or CVD risk reduction ${ }^{(2-6)}$.

At present, the primary lipid target for CVD risk reduction is LDL-cholesterol, with non-HDL-cholesterol and apoB as alternate targets. However, it has been suggested that nonHDL-cholesterol and apoB may be more relevant targets as
non-HDL-cholesterol contains all atherogenic cholesterol and there is one apoB on all atherogenic lipoprotein particles. Furthermore, both non-HDL-cholesterol and apoB have been shown to be highly correlated with CVD risk, especially when LDL-cholesterol appears to be within the normal range ${ }^{(7)}$, and have been added to the Third Report of the National Cholesterol Education Program - Adult Treatment Panel and the Canadian Cardiovascular Society (CCS) lipid guidelines as alternate lipid targets for CVD risk reduction ${ }^{(8,9)}$.

In contrast to the established relationship between oat $\beta$-glucan and LDL-cholesterol, there is currently little understanding of the relationship between oat $\beta$-glucan and alternate markers of CVD risk - that is, non-HDL-cholesterol and apoB. The objective of this study was to conduct a systematic review and meta-analysis of randomised-controlled trials (RCT) to analyse the evidence of the effect of oat $\beta$-glucan on

Abbreviations: MD, mean differences; MQS, Heyland Methodological Quality Score; RCT, randomised-controlled trials.

* Corresponding author: V. Vuksan, fax +1 416864 5538, email v.vuksan@utoronto.ca 
LDL-cholesterol, as well as for the first time on nonHDL-cholesterol and apoB, for CVD risk reduction.

\section{Methods \\ Protocol and registration}

The Cochrane Handbook for Systematic Reviews of Interventions was used to plan and conduct this meta-analysis ${ }^{(10)}$. Results were reported in accordance with the Preferred Reporting Items for Systematic Reviews and Meta-Analysis guidelines ${ }^{(11)}$. The review protocol is available online at ClinicalTrials.gov (registration no. NCT02068248).

\section{Search strategy and data sources}

MEDLINE, Embase, CINAHL and the Cochrane Central Register of Controlled Trials were searched, using the search strategy presented in the online Supplementary Table S1, through 5 November 2015, to identify RCT investigating the effects of oat $\beta$-glucan on LDL-cholesterol, non-HDL-cholesterol or apoB. Manual searches of references supplemented the electronic search. One unpublished trial from our group was included in the analysis ${ }^{(12)}$. No language restrictions were imposed.

\section{Study eligibility}

All titles and abstracts were initially assessed according to inclusion and exclusion criteria outlined in the online Supplementary Table S2. In brief, only RCT that investigated the effects of supplementing $\beta$-glucan from oat products on LDLcholesterol, non-HDL-cholesterol and/or apoB were included in the analysis ${ }^{(13,14)}$. Trials that did not report non-HDLcholesterol but provided enough information to permit the calculation of non-HDL-cholesterol (total cholesterol (TC) HDL-cholesterol) were also considered. Included trials involved any population, had a minimum follow-up period of 3 weeks, as per the United States Food and Drug Administration (US FDA $)^{(13,15)}$, administered any dose of $\beta$-glucan and provided enough information to calculate a treatment effect.

\section{Data extraction and quality assessment}

H. V. T. H. and A. Z. independently reviewed all studies that passed the initial assessment. A standardised proforma was used to extract relevant data including sample size, subject characteristics (health status, sex, age, weight, etc.), study setting (inpatient/outpatient), study design (parallel/crossover), follow-up duration, $\beta$-glucan dose, comparator, background diet, energy balance and funding source. If the $\beta$-glucan content was not reported, oat bran and whole oats were estimated at 6.9 and $5.0 \%{ }^{(16,17)} \beta$-glucan, respectively, and oat soluble fibre was estimated at $92.5 \% \beta$-glucan ${ }^{(18)}$. The mean and standard deviation values were extracted for LDL-cholesterol, non-HDL-cholesterol and apoB at baseline and follow-up for both control and intervention groups. When standard deviation values were not reported, they were derived from available data (95\% CI, $P$-values, $t$ or $F$ statistics, sEm) using standard formulae ${ }^{(10)}$. If available, mean change from baseline and standard deviation values for both groups, mean end difference and standard deviation values, and/or mean change from baseline difference and standard deviation values between groups were also extracted.

The Heyland Methodological Quality Score (MQS) was used to assess study quality ${ }^{(19)}$. Points were given on the basis of methods (randomisation, blinding and analysis), sample (selection, comparibility and follow-up) and intervention (protocol, co-intervention and cross-overs) and a maximum of 13 points could be received. Trials that received scores of $\geq 8$ were considered to be of higher quality.

The Cochrane Risk of Bias Tool was used to assess the study risk of bias ${ }^{(10)}$. Domains of bias assessed were sequence generation, allocation concealment, blinding, outcome data and outcome reporting. Trials were considered high risk when methodological flaws were likely to have affected the true outcome, low risk if the flaw was deemed inconsequential and unclear risk when insufficient information was provided to permit judgement. Authors were contacted for additional information where necessary. All disagreements on the MQS and Risk of Bias Tool were resolved by consensus.

\section{Data management and analysis}

Data were analysed using Review Manager (RevMan), version 5.3 (The Nordic Cochrane Centre, The Cochrane Collaboration), for primary analyses. The difference between the change from baseline values for the intervention and the control arms was derived from each trial for the end points of LDL-cholesterol, non-HDL-cholesterol and apoB. When non-HDL-cholesterol was not reported, it was calculated from aggregate data by subtracting HDL-cholesterol from TC. A previously developed formula was used to calculate SD for calculated values of non-HDL-cholesterol ${ }^{(20)}$. If change from baseline values were not available, end-of-treatment values were used. For trials containing multiple intervention or control arms, a weighted average was applied to combine them in order to create a single pair-wise comparison and to mitigate the unit-of-analysis error $^{(10)}$. Paired analyses were conducted for all cross-over studies $^{(21)}$. Where necessary, a pooled correlation coefficient was derived and used for calculation of an imputed sD for the between-treatment difference. Correlation coefficients between baseline and end-of-treatment values within each individual cross-over trial were derived from the reported within- and between-treatment SD according to a published formula ${ }^{(21)}$. These correlation coefficients were transformed into $z$-scores and SD, meta-analysed using inverse-variance weighing and back-transformed to derive the pooled correlation coefficient. For end points, when a pooled correlation coefficient for imputing missing SD could not be derived, a value of 0.50 was assumed, as it is a conservative estimate for an expected range of $0-1$. The values derived from each trial were pooled and analysed for LDL-cholesterol, non-HDL-cholesterol and apoB using the generic inverse-variance method with random effects models, which were used even in the absence of statistically significant between-study heterogeneity, as they yield more conservative summary effect estimates in the presence of 
residual heterogeneity. Data are expressed as mean differences (MD) with $95 \%$ CI. Furthermore, results are presented separately according to individual study inclusion criteria. The hypercholesterolic group included studies that recruited participants who were hypercholesterolaemic, and the unclassified group included studies that did not specify that participants had to be hypercholesterolaemic. A two-sided $P$-value $<0.05$ was set as the level of significance for comparisons of MD.

Inter-study heterogeneity was tested using Cochran's $Q$ statistic and quantified using the $I^{2}$-statistic with a significance level set at $P<0 \cdot 10 . I^{2}$ values $<50, \geq 50$ to $<75$ and $\geq 75 \%$ were considered to be evidence for 'moderate,' 'substantial' and 'considerable' heterogeneity, respectively ${ }^{(10)}$. Sources of heterogeneity were explored using sensitivity and subgroup analyses. To determine whether a single trial exerted undue influence on the overall results, sensitivity analyses were performed in which each individual trial was removed from the meta-analysis and the effect size was re-calculated with the remaining trials. Sensitivity analyses were also undertaken using correlation coefficients of $0 \cdot 25$, 0.50 and 0.75 to determine whether the overall results were robust to the use of different derived correlation coefficients in paired analyses of cross-over trials. A priori subgroup analyses (continuous and categorical) were conducted for baseline values of LDL-cholesterol, non-HDL-cholesterol and apoB within the intervention arm, dose, design, follow-up and study quality. Metaregression was performed to assess the significance of subgroup effects with STATA software, version 13 (StataCorp LP), with a significance level set at $P<0.05$.

Publication bias was investigated by visual inspection of funnel plots and quantitatively assessed using Egger's and Begg's tests, where $P<0.05$ was considered evidence for small study effects.

Funnel plots were used to display the relative treatment effect and its $95 \%$ CI for each trial and dose amount and for the overall random-effects meta-analyses.

\section{Results}

\section{Search results}

The search strategy initially yielded 8190 publications, of which 269 were reviewed in full and fifty-eight ( $n$ 3974) were included in the final meta-analysis (Fig. 1). In total, fifty-six trials reported data on LDL-cholesterol ( $n$ 3745) and seventeen on apoB ( $n$ 1070). Only one trial reported data on non-HDL-cholesterol; however, fifty-six other trials reported enough information to calculate non-HDL-cholesterol ( $n$ 3926).

\section{Trial characteristics}

The characteristics of the included trials are summarised in Table 1. Trials were conducted in both in-patient and outpatient settings with twenty-five in North American (nineteen in USA, five in Canada and one in Mexico), nineteen in Europe (six in Sweden, four in England, three in the Netherlands, two in France and one each in Denmark, Finland, Germany and Greece), eight in Australia and New Zealand, three in Asia (two in China and one in Thailand), one in South America
(Venezuela) and one in the Middle East (Iran). All trials were randomised, with $66 \%$ (thirty-eight trials) utilising a parallel design and $34 \%$ (twenty trials) utilising a cross-over design. Participants were generally middle aged (median age $=50 \cdot 6$ (range: 10-67) years) with an approximately equal number of men and women. Participants were slightly overweight (median BMI $=26 \cdot 8$ (range: $22 \cdot 8-32 \cdot 2$ ) $\mathrm{kg} / \mathrm{m}^{2}$ ), despite only 4 four trials recruiting on the basis of overweight/obese. Two-thirds of the trials (thirty-nine trials) were conducted in hypercholesterolaemic individuals. The dose of oat $\beta$-glucan ranged from 0.9 to $10.3 \mathrm{~g} / \mathrm{d}$ with a median dose of $3.5 \mathrm{~g} / \mathrm{d}$. Treatment duration ranged from 3 to 12 weeks with the median length being 6 weeks for trials reporting LDL-cholesterol and nonHDL-cholesterol and 5 weeks for trials reporting apoB.

Very few studies (nine trials, 16\%) were considered to be of higher quality $(\mathrm{MQS} \geq 8$ ). Lack of or poor description of randomisation, patient selection, protocol analysis and absence of double-blinding contributed to lower scores (online Supplementary Table S3). The Cochrane Risk of Bias Tool (online Supplementary Fig. S1 and Table S4) showed that seventeen trials (29\%) had low risk of bias and forty-two trials (71\%) had unclear risk of bias for random sequence generation. A total of thirteen trials (22\%) had low risk of bias, and forty-six trials (78\%) were unclear for allocation concealment. Moreover, thirty trials (50\%) had high risk of bias, twenty-one trials (36\%) had low risk of bias and eight trials (14\%) had unclear performance bias (blinding of participants and personnel); five trials ( $8 \%$ ) has high risk of bias, forty-nine trials ( $84 \%$ ) had low risk of bias and five trials ( $8 \%$ ) had unclear risk of bias for attrition bias. The majority of trials (93\%) had low risk of bias for reporting bias, whereas the remainder of the trials $(7 \%)$ had unclear risk of bias for these items. Funding of trials included agency (26\%), agency-industry (16\%), industry (34\%) sources or were not reported (24\%).

\section{Effect on LDL-cholesterol}

The effect of oat $\beta$-glucan on LDL-cholesterol is shown in Fig. 2. Overall, a significant LDL-cholesterol reduction was observed with a median dose of $3.5 \mathrm{~g} / \mathrm{d}$ for a median duration of 6 weeks (MD $=-0.19 \mathrm{mmol} / \mathrm{l} ; \quad 95 \% \quad$ CI $-0.23, \quad-0.14 ; \quad P<0.00001)$. However, substantial evidence of inter-study heterogeneity was present in the overall analysis $\left(I^{2}=79 \%\right.$; $\left.P<0.00001\right)$. Systematic removal of individual trials did not alter the results.

Categorical a priori subgroup analyses revealed that the LDL-cholesterol lowering effect of oat $\beta$-glucan was modified by both study design (between-group $\mathrm{MD}=0.09 \mathrm{mmol} / \mathrm{l} ; 95 \% \mathrm{CI}$ $0.01,0.17 ; P=0.03)$ - studies that utilised a cross-over design demonstrated an MD of $-0.25 \mathrm{mmol} / 1$ (95\% CI $-0.31,-0 \cdot 18)$, whereas studies that utilised a parallel design showed an MD of $-0.16 \mathrm{mmol} / \mathrm{l} \quad(95 \% \mathrm{CI}-0.20,-0 \cdot 11)$ - and study duration (between-group $\mathrm{MD}=0.09 \mathrm{mmol} / 1 ; 95 \% \mathrm{CI} 0.02,0.17 ; P=0.03$ ) studies where oat $\beta$-glucan was administered for $<6$ weeks demonstrated an MD of $-0.24 \mathrm{mmol} / 1$ (95\% CI $-0 \cdot 29,-0 \cdot 18)$, whereas studies that administered oat $\beta$-glucan for 6 weeks or more showed an MD of $-0.15 \mathrm{mmol} / 1$ (95\% CI $-0.20,-0.09)$, (online Supplementary Fig. S2). Continuous meta-regression analyses demonstrated an inverse association between baseline 


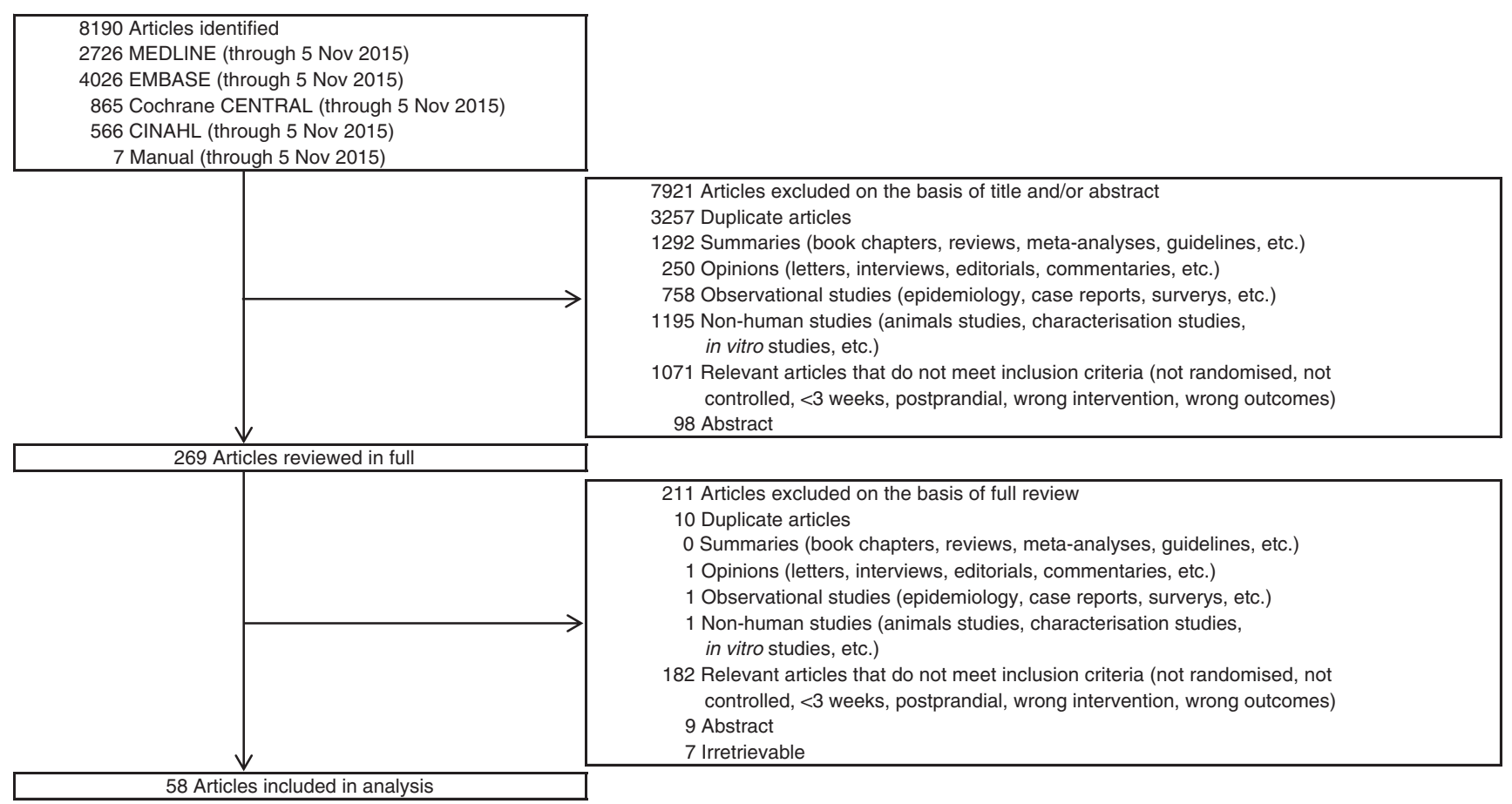

Fig. 1. Flow of literature. Summary of search and selection process.

LDL-cholesterol and treatment differences for LDL-cholesterol ( $\beta=-0.09 \mathrm{mmol} / 1 ; \quad 95 \%$ CI $-0.15, \quad-0.03 ; \quad P=0.004$ ) (online Supplementary Table S5). Heterogeneity remained significant, and could not be explained by subgroup analyses.

\section{Effect on non-HDL-cholesterol}

The effect of oat $\beta$-glucan on non-HDL-cholesterol is shown in Fig. 3. Overall, non-HDL-cholesterol was significantly reduced by $-0.20 \mathrm{mmol} / 1(95 \% \mathrm{CI}-0 \cdot 26,-0 \cdot 15), P<0 \cdot 00001$, with a median dose of $3.5 \mathrm{~g} / \mathrm{d}$ for a median duration of 6 weeks. Considerable evidence of inter-study heterogeneity was present in the overall analysis $\left(I^{2}=99 \% ; P<0 \cdot 00001\right)$. Systematic removal of individual trials did not alter the results.

Categorical a priori subgroup analyses revealed that the nonHDL-cholesterol lowering was not modified by dose, study duration, study design, MQS scores or baseline non-HDLcholesterol levels (online Supplementary Fig. S3). Furthermore, continuous meta-regression analyses did not reveal associations between dose, treatment duration or baseline non-HDLcholesterol levels (online Supplementary Table S5).

\section{Effect on apoB}

The effect of oat $\beta$-glucan on apoB is shown in Fig. 4. Overall, there was evidence of a significant lowering of apoB with a median dose of $3.5 \mathrm{~g} / \mathrm{d}$ for a median duration of 5 weeks $(\mathrm{MD}=-0.03 \mathrm{~g} / \mathrm{l} ; \quad 95 \%$ CI $-0.05, \quad-0.02 ; \quad P<0.0001)$ with moderate evidence of heterogeneity $\left(I^{2}=38 \% ; \quad P=0.06\right)$. Systematic removal of individual trials did not alter the results.

Categorical a priori subgroup analyses revealed that the apoB lowering by oat $\beta$-glucan was not modified by dose, study duration, study design, MQS scores or baseline apoB levels (online Supplementary Fig. S4). Furthermore, continuous meta-regression analyses did not reveal associations between dose, treatment duration or baseline apoB levels (online Supplementary Table S5).

\section{Publication bias}

Funnel plots for LDL-cholesterol, non-HDL-cholesterol and apoB are shown in Fig. 5. Visual inspection of funnel plots suggested minor asymmetry in the LDL-cholesterol and nonHDL-cholesterol analyses, with tendencies for the publication of small and/or imprecise trials favouring oat $\beta$-glucan for both. This was confirmed by Begg's tests $(P=0.061)$ for LDLcholesterol; however, neither Egger's $(P=0.381)$ nor Begg's $(P=0 \cdot 528)$ test was significant for non-HDL-cholesterol.

\section{Discussion}

The present systematic review and meta-analysis of fifty-eight trials involving 3974 participants assessed the effects of oat $\beta$-glucan on clinical lipid targets for CVD risk reduction (LDLcholesterol, non-HDL-cholesterol and apoB). Diets enriched with a median dose of $3.5 \mathrm{~g} / \mathrm{d}$ of $\beta$-glucan were found to modestly improve LDL-cholesterol (-4.2\%), non-HDLcholesterol $(-4 \cdot 8 \%)$ and apoB $(-2 \cdot 3 \%)$, compared with control diets.

Brown et al. ${ }^{(79)}$ were the first to undertake a comprehensive meta-analysis of all viscous, soluble fibre types on cholesterol. Although the main objective was to study the cholesterollowering effect of all viscous, soluble fibre types, it was, nevertheless, the first to consolidate data on oats and 


\section{N British Journal of Nutrition}

\begin{tabular}{|c|c|c|c|c|c|c|c|c|c|c|c|}
\hline Reference (study, year) $\dagger$ & Participantsł & Age (years) & $\begin{array}{c}\mathrm{BMI} \\
\left(\mathrm{kg} / \mathrm{m}^{2}\right)\end{array}$ & Design & Blinding & Dose§ $(g / d)$ & Comparator & Background diet & MQS\| & $\begin{array}{l}\text { Funding } \\
\text { source } \Uparrow\end{array}$ & Setting \\
\hline \multicolumn{12}{|l|}{ Hypercholesterolaemic trials } \\
\hline Amundsen et al., 2003 & 16 (9M:7F) & 57.0 & $25 \cdot 4$ & C, 3 weeks & SB & $5 \cdot 1$ & Nothing & AHA step I & 6 & $A-I$ & OP, Sweden \\
\hline Anderson et al., $1991^{(23)}$ & 20 (20M:OF) & $61 \cdot 0$ & & P, 3 weeks & NB & & & Typical & 4 & A & IP, USA \\
\hline Control & 10 & $65 \cdot 0$ & 25.6 & & & & Wheat & American diet & & & \\
\hline Oat bran & 10 & 57.0 & 25.6 & & & $12 \cdot 4$ & & & & & \\
\hline Berg et al., $2003^{(24)}$ & 235 (235M:OF) & & & P, 4 weeks & NB & $2-3.5$ & & NCEP step 2 & 7 & $N / R$ & IP, Germany \\
\hline Control & 136 & $54 \cdot 0$ & 30.1 & & & & Nothing & & & & \\
\hline Oat bran & 99 & 52.9 & 30.1 & & & & & & & & \\
\hline Biorklund et al., 2005 & 54 & & & P, 5 weeks & SB & & & None & 7 & A & OP, Sweden \\
\hline Control & 20 (10M:10F) & & & & & & Rice & & & & Netherlands \\
\hline Oat bran & 19 (10M:9F) & & & & & $5 \cdot 0$ & & & & & \\
\hline Oat bran & 15 (8M:7F) & & & & & $10 \cdot 0$ & & & & & \\
\hline Biorklund et al., 2008 & 43 (19M:24F) & 58.0 & $25 \cdot 0$ & P, 5 weeks & SB & & & None & 8 & A & OP, Sweden \\
\hline Control & 21 & & & & & & Maltodextrin & & & & \\
\hline Oat concentrate & 22 & & & & & 4.0 & & & & & \\
\hline \multirow{3}{*}{ Braaten et al., $1994^{(27)}$} & 19 & & & C, 4 weeks & SB & 5.8 & Maltodextrin & None & 5 & 1 & OP, Canada \\
\hline & $9 \mathrm{M}$ & 52.0 & $26 \cdot 0$ & & & & & & & & \\
\hline & $10 \mathrm{~F}$ & 56.0 & $26 \cdot 3$ & & & & & & & & \\
\hline Bremer et al., $1991^{(28)}$ & 12 (5M:7F) & 53.0 & & C, 4 weeks & SB & $3 \cdot 1$ & Wheat & AHA step II & 7 & $A-I$ & OP, New Zealand \\
\hline Charlton et al., 2012 & 87 & $51 \cdot 0$ & $27 \cdot 3$ & $\mathrm{P}, 6$ weeks & SB & & & $\begin{array}{l}\text { Australian guide to } \\
\text { healthy eating }\end{array}$ & 9 & 1 & OP, Australia \\
\hline Control & 31 (15M:16F) & 49.8 & $27 \cdot 7$ & & & & Maize, rice & & & & \\
\hline Whole oats & 26 (11M:15F) & 51.9 & $27 \cdot 3$ & & & 1.5 & & & & & \\
\hline Whole oats & 30 (15M:15F) & $52 \cdot 4$ & 26.7 & & & 3.2 & & & & & \\
\hline Davidson et al., 1991 & 141 & & & $\mathrm{P}$, weeks & SB & & & NCEP step I & 5 & 1 & OP, USA \\
\hline Control & 15 (10M:5F) & $53 \cdot 1$ & $25 \cdot 8$ & & & & Wheat & & & & \\
\hline Whole oats & 20 (7M:13F) & $51 \cdot 1$ & $26 \cdot 2$ & & & 1.2 & & & & & \\
\hline Oat bran & 23 (12M:10F) & 51.6 & 24.6 & & & $2 \cdot 0$ & & & & & \\
\hline Whole oats & 21 (15M:7F) & 55.0 & $26 \cdot 1$ & & & 2.4 & & & & & \\
\hline Oat bran & 20 (14M:5F) & 52.6 & 24.8 & & & 4.0 & & & & & \\
\hline Whole oats & 21 (9M:11F) & $51 \cdot 0$ & $25 \cdot 2$ & & & 3.6 & & & & & \\
\hline Oat bran & 21 (13M:9F) & 54.8 & 25.0 & & & $6 \cdot 0$ & & & & & \\
\hline $\begin{array}{l}\text { Demark-Wahnefried et al., } \\
1990^{(31)}\end{array}$ & 35 & & & $\mathrm{P}, 12$ weeks & NB & & & Low fat, low & 5 & $A-I$ & OP, USA \\
\hline Control & 16 & & & & & & Nothing & Cholesterol & & & \\
\hline Oat bran & 19 & & & & & 3.5 & & & & & \\
\hline Johnston et al., 1998 ${ }^{(32)}$ & 124 & & & P, 6 weeks & DB & & & None & 6 & 1 & OP, USA \\
\hline Control & 62 (38M:24F) & 57.3 & & & & & Maize & & & & \\
\hline Whole oats & 62 (40M:22F) & 56.7 & & & & 2.8 & & & & & \\
\hline Karmally et al., $2005^{(33)}$ & 152 & & & P, 6 weeks & NB & & & NCEP step I & 3 & 1 & OP, USA \\
\hline Control & 79 (21M:58F) & 48.9 & 28.5 & & & & Maize & & & & \\
\hline Whole oats & 73 (28M:45F) & 49.1 & 29.9 & & & $2 \cdot 8$ & & & & & \\
\hline Kerckhoffs et al., $2003^{(34)}$ & 48 (21M:27F) & 51.3 & 24.9 & P, 4 weeks & NB & & & None & 6 & $N / R$ & OP, Netherlands \\
\hline Control & 23 & & & & & & Wheat & & & & \\
\hline Oat bran/concentrate & 25 & & & & & $5 \cdot 9$ & & & & & \\
\hline Kestin et al., 1990(35) & 24 (24M:OF) & $46 \cdot 0$ & $25 \cdot 4$ & C, 4 weeks & NB & 5.0 & Wheat & Low-fibre diet & 6 & I & OP, Australia \\
\hline Leadbetter et al., $1991^{(36)}$ & 40 (20M:20F) & & 26.8 & C, 4 weeks & NB & $2 \cdot 1,4 \cdot 2,6 \cdot 2$ & Nothing & None & 8 & $i$ & OP, New Zealand \\
\hline Lepre \& Crane, $1992^{(37) *}$ & 37 & 51.9 & $25 \cdot 1$ & C, 8 weeks & DB & 3.0 & Wheat & Customised & 6 & $N / R$ & OP, Australia \\
\hline Liatis et al., $2009^{(38)}$ & 41 & & & P, 3 weeks & $\mathrm{DB}$ & & & None & 7 & 1 & OP, Greece \\
\hline Control & 18 (11M:7F) & 66.5 & $27 \cdot 0$ & & & & Wheat & & & & \\
\hline Whole oats & 23 (12M:11F) & 60.2 & 29.6 & & & 3.0 & & & & & \\
\hline Lovegrove et al., $2000^{(39)}$ & 62 & & & P, 8 weeks & DB & & & None & 7 & $N / R$ & OP, UK \\
\hline Control & 31 (16M:15F) & 56.8 & $25 \cdot 8$ & & & & Wheat & & & & \\
\hline Oat concentrate & 31 (15M:16F) & $56 \cdot 3$ & 26.0 & & & 3.0 & & & & & \\
\hline Maki et al., $2003^{(40)}$ & 18 (13M:5F) & 10.6 & $27 \cdot 4$ & C, 4 weeks & DB & $2 \cdot 8$ & RTE cereal & NCEP step I & 6 & 1 & OP, USA \\
\hline
\end{tabular}


N British Journal of Nutrition

\begin{tabular}{|c|c|c|c|c|c|c|c|c|c|c|c|}
\hline Reference (study, year) $\dagger$ & Participantsł & Age (years) & $\begin{array}{c}\mathrm{BMI} \\
\left(\mathrm{kg} / \mathrm{m}^{2}\right)\end{array}$ & Design & Blinding & Dose§ (g/d) & Comparator & Background diet & MQS\| & $\begin{array}{l}\text { Funding } \\
\text { source } \|\end{array}$ & Setting \\
\hline Maki et al., 2010(41) & 144 & & & $\mathrm{P}, 12$ weeks & NB & & & None & 4 & 1 & OP, USA \\
\hline Control & 67 (12M:55F) & 47.5 & $32 \cdot 2$ & & & & Maize, wheat & & & & \\
\hline Whole oats & 77 (19M:58F) & 50.1 & $32 \cdot 0$ & & & 3.0 & & & & & \\
\hline Mårtensson et al., 2005(42) & 56 & & & P, 5 weeks & DB & & & None & 6 & $A-I$ & OP, Sweden \\
\hline Control & 18 (7M:11F) & 56.0 & $25 \cdot 2$ & & & & Dairy-based & & & & \\
\hline Oat bran & 20 (9M:11F) & 55.0 & $26 \cdot 0$ & & & $3 \cdot 0$ & Concentrate & & & & \\
\hline Oat bran & 18 (8M:10F) & 56.0 & 24.5 & & & 3.6 & & & & & \\
\hline Momenizadeh et al., 2014(43) & 60 (21M:39F) & 51.1 & & $\mathrm{P}, 6$ weeks & NB & & & None & 7 & $N / R$ & OP, Iran \\
\hline Control & & & 29.0 & & & & Wheat & & & & \\
\hline Oat bran & & & 28.9 & & & $2 \cdot 1$ & & & & & \\
\hline Noakes et al., $1996^{(44)}$ & 23 (13M:10F) & $51 \cdot 0$ & 29.0 & C, 4 weeks & NB & $12 \cdot 3$ & $\begin{array}{l}\text { Resistant } \\
\text { Starch }\end{array}$ & $\begin{array}{l}\text { Customised } \\
\text { Low-fat, low-fibre } \\
\text { diet }\end{array}$ & 3 & $N / R$ & OP, Australia \\
\hline Onning et al., $1999^{(45)}$ & 52 & 62.6 & $27 \cdot 1$ & C, 5 weeks & DB & 3.8 & Rice & None & 6 & A & OP, Sweden \\
\hline Panahi, $2006^{(12)}$ & 105 (56M:49F) & $62 \cdot 2$ & $25 \cdot 7$ & P, 6 weeks & DB & & & NCEP step II & 10 & $N / R$ & OP, Canada \\
\hline Control & 35 & & & & & & Wheat, rice & & & & \\
\hline Oat concentrate & 35 & & & & & 3.0 & & & & & \\
\hline Oat concentrate & 35 & & & & & 9.0 & & & & & \\
\hline Queenan et al., 2007 $7^{(46)}$ & 75 & & & P, 6 weeks & DB & & & None & 7 & A & OP, USA \\
\hline Control & 40 (12M:28F) & $45 \cdot 3$ & & & & & Dextrose & & & & \\
\hline Oat concentrate & 35 (13M:22F) & 44.5 & & & & $6 \cdot 0$ & & & & & \\
\hline Reyna-Villasmil et al., $2007^{(47)}$ & 38 (38M:OF) & 59.8 & & P, 8 weeks & NB & & & AHA step II & 6 & $N / R$ & OP, Venezuela \\
\hline Control & 19 & & 28.2 & & & & Wheat & & & & \\
\hline Oat concentrate & 19 & & 28.4 & & & $6 \cdot 0$ & & & & & \\
\hline Reynolds et al., 2000 ${ }^{(48)}$ & 43 (21M:22F) & & & $P, 4$ weeks & DB & & & AHA step I & 7 & $N / R$ & OP, USA \\
\hline Control & & & & & & & Maize & & & & \\
\hline Whole oats & & & & & & $2 \cdot 5$ & & & & & \\
\hline Romero et al., $1998^{(49)}$ & 20 & & & P, 8 weeks & NB & & & None & 4 & $N / R$ & OP, Mexico \\
\hline Control & 10 & 36.0 & 26.6 & & & & Wheat & & & & \\
\hline Oat bran & 10 & 38.0 & 27.1 & & & $2 \cdot 6$ & & & & & \\
\hline Stewart et al., $1992^{(50)}$ & 24 (11M:13F) & 46.0 & 23.5 & C, 6 weeks & NB & 3.5 & Nothing & Low fat & 5 & I & OP, New Zealand \\
\hline Theuwissen \& Mensink, $2007^{(51) *}$ & 42 (20M:22F) & 52.4 & $25 \cdot 0$ & C, 4 weeks & $\mathrm{DB}$ & $5 \cdot 0$ & Wheat & None & 7 & 1 & OP, Netherlands \\
\hline Thongoun et al., 2013 ${ }^{(52)}$ & $24(2 \mathrm{M}: 22 \mathrm{~F})$ & $51 \cdot 0$ & 26.8 & C, 4 weeks & NB & 3.5 & Rice & None & 8 & $N / R$ & OP, Thailand \\
\hline Turnbull \& Leeds, $1987^{(53)}$ & $17(9 \mathrm{M}: 8 \mathrm{~F})$ & & & C, 4 weeks & NB & $6 \cdot 3$ & Wheat & None & 9 & 1 & OP, UK \\
\hline Uusitupa et al., $1992^{(54)}$ & 36 & & & P, 8 weeks & DB & & & None & 5 & $A-I$ & OP, Finland \\
\hline Control & 16 (10M:6F) & $45 \cdot 0$ & $26 \cdot 7$ & & & & Wheat & & & & \\
\hline Oat bran & 20 (10M:10F) & 50.0 & $26 \cdot 3$ & & & $10 \cdot 3$ & & & & & \\
\hline Van Horn et al., 1991 & 80 & & & P, 8 weeks & NB & & & None & 4 & I & OP, USA \\
\hline Control & 38 (19M:19F) & $42 \cdot 1$ & $26 \cdot 2$ & & & & Nothing & & & & \\
\hline Whole oats & 42 (21M:21F) & 42.9 & $26 \cdot 2$ & & & $2 \cdot 0$ & & & & & \\
\hline Van Horn et al., 2001 ${ }^{(56)}$ & 64 & & & P, 6 weeks & NB & & & NCEP step I & 6 & I & OP, USA \\
\hline Control & 32 & 67.3 & 26.6 & & & & Wheat & & & & \\
\hline Whole oats & 32 & 65.0 & 26.8 & & & 1.9 & & & & & \\
\hline Whyte et al., $1992^{(57)}$ & 23 (23M:OF) & $45 \cdot 0$ & 25.5 & C, 4 weeks & NB & 8.5 & Wheat & Australian diet & 6 & 1 & OP, USA \\
\hline Wolever et al., 2010 ${ }^{(58) \star *}$ & 367 & & & P, 4 weeks & DB & & & None & 10 & A-I & OP, Canada \\
\hline Control & 87 (36M:51F) & $52 \cdot 0$ & 28.0 & & & & Wheat & & & & \\
\hline High MW oat bran & 86 (43M:43F) & $52 \cdot 0$ & $27 \cdot 3$ & & & 3.0 & & & & & \\
\hline Medium MW oat bran & 64 (27M:37F) & 52.0 & 26.9 & & & 3.0 & & & & & \\
\hline Medium MW oat bran & 67 (33M:34F) & $52 \cdot 0$ & 27.9 & & & 4.0 & & & & & \\
\hline Low MW oat bran & 63 (22M:41F) & 53.0 & 27.5 & & & 4.0 & & & & & \\
\hline Zhang et al., $2012^{(59)}$ & 166 & & & P, 6 weeks & NB & & & None & 4 & A & OP, China \\
\hline Control & 81 (32M:49) & 53.7 & 25.5 & & & & Wheat & & & & \\
\hline Whole oats & 85 (33M:52F) & $52 \cdot 7$ & 25.5 & & & $3 \cdot 3$ & & & & & \\
\hline Unclassified trials & & & & & & & & & & & \\
\hline Beck et al., 2010 ${ }^{(60)}$ & 56 (OM:56F) & & & P, 12 weeks & SB & & & None & 6 & A & OP, Australia \\
\hline Control & 16 & 37.1 & 29.2 & & & & Nothing & & & & \\
\hline Oat bran & 21 & 37.7 & 29.3 & & & $5.0-6.0$ & & & & & \\
\hline Oat bran & 19 & 37.4 & 29.3 & & & $8 \cdot 0-9.0$ & & & & & \\
\hline
\end{tabular}


N British Journal of Nutrition

\begin{tabular}{|c|c|c|c|c|c|c|c|c|c|c|c|}
\hline Reference (study, year) $\dagger$ & Participantsł & Age (years) & $\begin{array}{c}\mathrm{BMI} \\
\left(\mathrm{kg} / \mathrm{m}^{2}\right)\end{array}$ & Design & Blinding & Dose§ (g/d) & Comparator & Background diet & MQS॥ & $\begin{array}{l}\text { Funding } \\
\text { sourceף }\end{array}$ & Setting \\
\hline Chen et al., 2006 ${ }^{(61) \star}$ & 110 & & & P, 12 weeks & DB & & & None & 10 & $A-I$ & OP, USA \\
\hline Control & 56 (22M:34F) & $46 \cdot 1$ & $29 \cdot 3$ & & & & Wheat, maize & & & & \\
\hline Oat bran & 54 (22M:32F) & 49.7 & 28.5 & & & 7.4 & & & & & \\
\hline Cugnet-Anceau et al., 2010 (62) & 53 & & & P, 8 weeks & DB & & & None & 5 & A & OP, France and Sweden \\
\hline Control & 24 & $61 \cdot 8$ & $29 \cdot 0$ & & & & Maltodextrin & & & & \\
\hline Oat concentrate & 29 & 61.9 & 30.5 & & & 3.5 & & & & & \\
\hline Davy et al., 2002 ${ }^{(63)}$ & 36 & & & $\mathrm{P}, 12$ weeks & NB & & & None & 5 & $N / R$ & OP, USA \\
\hline Control & 18 & $61 \cdot 0$ & $29 \cdot 2$ & & & & Wheat & & & & \\
\hline Oat bran/whole oats & 18 & 57.0 & 29.6 & & & 5.5 & & & & & \\
\hline Gerhardt \& Gallo, $1998^{(64)}$ & 27 & 51.7 & & P, 6 weeks & DB & & & None & 6 & $N / R$ & OP, USA \\
\hline Control & 14 & & & & & & Rice & & & & \\
\hline Oat bran & 13 & & & & & 3.1 & & & & & \\
\hline Gold \& Davidson, $1988^{(65)}$ & 44 & & & P, 4 weeks & DB & & & None & 5 & A & OP, USA \\
\hline Control & 25 & & & & & & Wheat & & & & \\
\hline Oat bran & 19 (15M:4F) & $26 \cdot 1$ & & & & 2.3 & & & & & \\
\hline Ibrugger et al., $2013^{(66)}$ & 13 (6M:7F) & 22.9 & 22.8 & C, 3 weeks & SB & 3.3 & Nothing & None & 7 & A & OP, Denmark \\
\hline Kabir et al., $2002^{(67)}$ & 13 (13M:OF) & 58.4 & 27.5 & C, 4 weeks & NB & $\begin{array}{l}3.0 \\
3.0\end{array}$ & Wheat & None & 8 & $A-1$ & OP, France \\
\hline Ma et al., $2013^{(68)}$ & 197 & & & P, 4 weeks & NB & & & $\begin{array}{l}\text { Nutrition guidelines } \\
\text { for Chinese } \\
\text { residents }\end{array}$ & 5 & 1 & IP, China \\
\hline Control & 61 (28M:33F) & $59 \cdot 3$ & $26 \cdot 8$ & & & & Nothing & & & & \\
\hline Whole oats & 65 (27M:38F) & 59.4 & $\begin{array}{l}26.0 \\
26.6\end{array}$ & & & 2.5 & & & & & \\
\hline Whole oats & 71 (26M:45F) & 60.3 & 26.9 & & & 5.0 & & & & & \\
\hline McGeoch et al., $2013^{(69)}$ & 27 (18M:9F) & 60.9 & 31.5 & C, 8 weeks & NB & 6.0 & Nothing & $\begin{array}{l}\text { Standard dietary } \\
\text { advice }\end{array}$ & 5 & A & OP, UK \\
\hline $\begin{array}{l}\text { Naumann et al., 2006 } \\
\text { Control }\end{array}$ & 47 (18M:29F) & $51 \cdot 7$ & $24 \cdot 2$ & P, 5 weeks & DB & & Rice & $\begin{array}{l}\text { advice } \\
\text { None }\end{array}$ & 6 & A & OP, Netherlands \\
\hline Oat concentrate & & & & & & 5.0 & & & & & \\
\hline Pick et al., $1996^{(71)}$ & 8 & $45 \cdot 5$ & $27 \cdot 6$ & C, 12 weeks & NB & 8.3 & White & Individualised & 5 & 1 & OP, Canada \\
\hline Pins et al., 2002 & $\begin{array}{r}0 \\
88\end{array}$ & & & P, 12 weeks & SB & & & None & 6 & 1 & OP, USA \\
\hline Control & 43 (22M:21F) & 46.4 & 30.6 & & & & Wheat & & & & \\
\hline Whole oats & 45 (23M:22F) & 48.7 & 31.2 & & & 5.4 & & & & & \\
\hline Poulter et al., 1994 $4^{(73)}$ & 59 (17M:42F) & $\begin{array}{l}40.7 \\
56.4\end{array}$ & & C. 4 weeks & NB & 2.0 & Nothing & None & 5 & 1 & OP, UK \\
\hline Robitaille et al., 2005 & 34 (OM:34F) & & & P, 4 weeks & NB & & & NCEP step I & 5 & A & OP, Canada \\
\hline Control & 16 & 37.4 & 29.5 & & & & Nothing & & & & \\
\hline Oat bran & 18 & $\begin{array}{l}3 / .4 \\
39.1\end{array}$ & $\begin{array}{l}28.5 \\
28.8\end{array}$ & & & 2.3 & & & & & \\
\hline Romero et al., $1998^{(49)}$ & 26 & & & P, 8 weeks & NB & & Wheat & None & 4 & $N / R$ & OP, Mexico \\
\hline Control & 14 & 29.0 & $26 \cdot 3$ & & & & & & & & \\
\hline Oat bran & 12 & 40.0 & 27.5 & & & 2.6 & & & & & \\
\hline Saltzman et al., 2001 ${ }^{(75)}$ & 43 & & & P, 6 weeks & NB & & & None & 8 & A-I & OP, USA \\
\hline Control & 21 (9M:12F) & 44.1 & 26.7 & & & & Nothing & & & & \\
\hline Whole oats & 22 (11M:11F) & 45.1 & $26 \cdot 1$ & & & 4.1 & & & & & \\
\hline Swain et al., $1990^{(76)}$ & 20 (4M:16F) & 30.0 & & C. 6 weeks & DB & $\begin{array}{l}4.1 \\
6.9\end{array}$ & Wheat & None & 6 & A & OP USA \\
\hline Van Horn et al., $1988^{(77)}$ & 236 & 42.4 & & P, 8 weeks & NB & & & AHA step I & 2 & $\mathrm{~N} / \mathrm{R}$ & OP, USA \\
\hline Control & 123 (45M:78F) & & & & & & Nothing & & & & \\
\hline Whole oats & 113 (41M:72F) & & & & & 2.8 & & & & & \\
\hline Zhang et al., $1992^{(78)}$ & 9 (7M:2F) & 55.1 & & C, 3 weeks & NB & 8.1 & Nothing & None & 5 & A & OP, Sweden \\
\hline
\end{tabular}

Cholesterol Education Program; N/R, not reported; I, industry; DB, double blind; RTE, ready to eat; MW, molecular weight.

† Whole oats can be oatmeal, instant oats, oat flakes or whole oat flour.

¥ The number of participants listed for each trial is the number of participants that completed the trial, and therefore the number used in our analyses and the number used for the reported baseline data (age and BMI), unless otherwise

indicated with "*'.

II Trials with an MQS $\geq 8$ were considered to be of higher quality.

I Agency funding is that from government, university or not-for-profit health agency sources. 


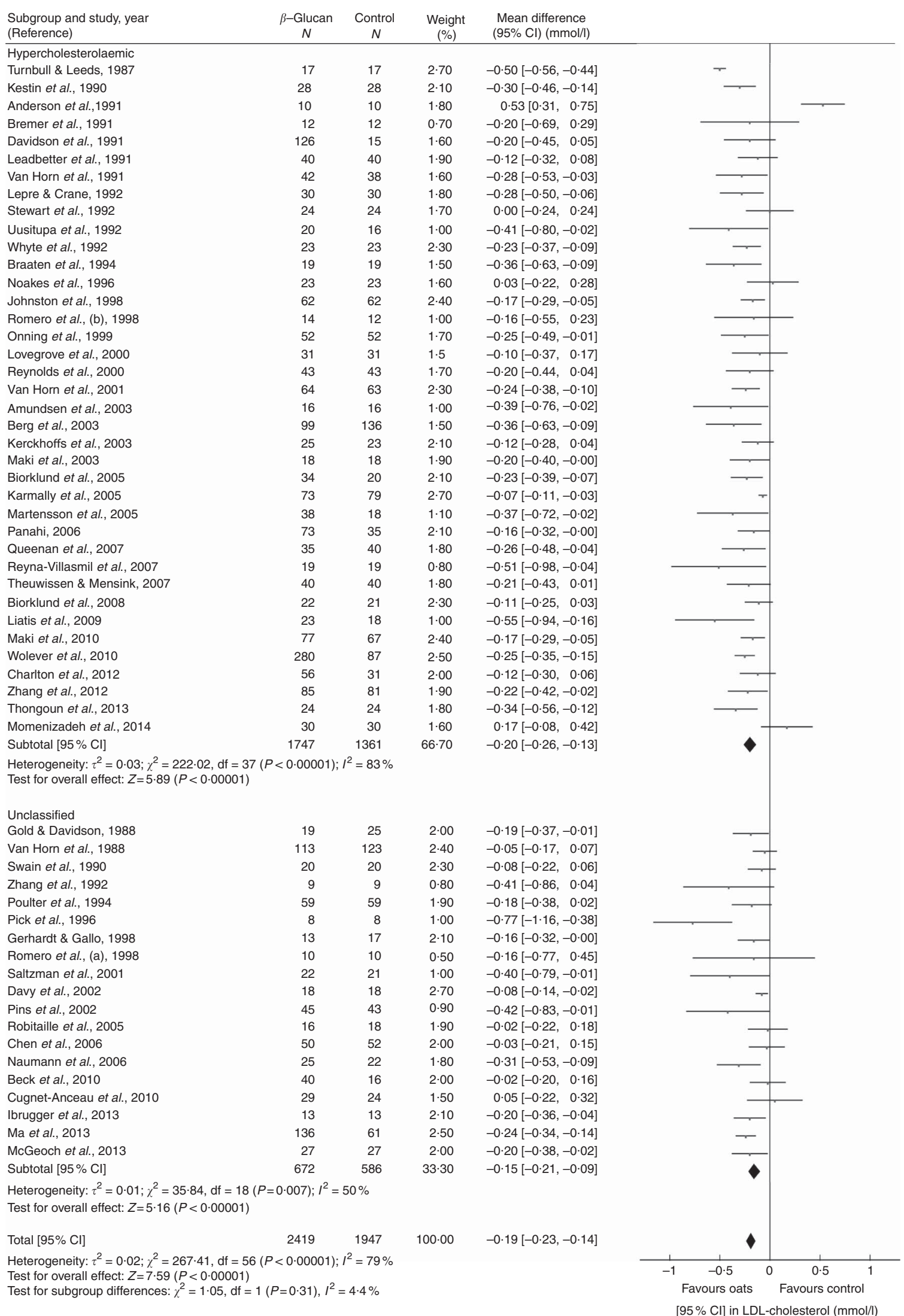

Fig. 2. Forest plot of randomised-controlled trials investigating the effect of oat $\beta$-glucan on LDL-cholesterol. Pooled effect estimate ( $(\mathrm{mmol} / \mathrm{l})$. Values are mean differences (MD) with $95 \% \mathrm{Cl}$, using the generic inverse-variance random effects models. Inter-study heterogeneity was quantified by $I^{2}$ at a significance of $P<0.10 . N$, number of participants in each treatment group. 


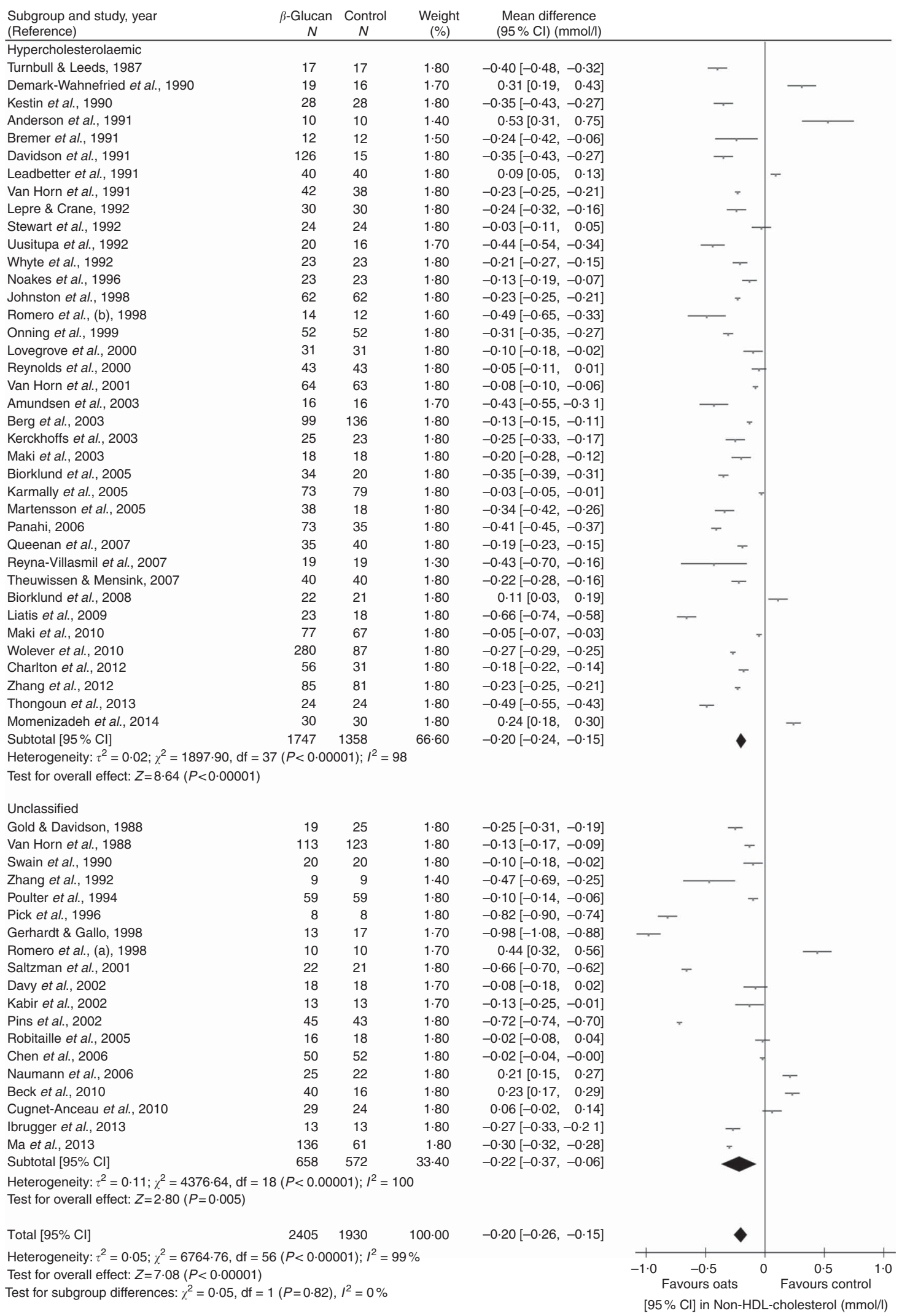

Fig. 3. Forest plot of randomised-controlled trials investigating the effect of oat $\beta$-glucan on non-HDL-cholesterol. Pooled effect estimate ( $\longrightarrow$ ) for non-HDLcholesterol $(\mathrm{mmol} / \mathrm{l})$. Values are mean differences (MD) with $95 \% \mathrm{Cl}$, using the generic inverse-variance random effects models. Inter-study heterogeneity was quantified by $I^{2}$ at a significance of $P<0 \cdot 10 . N$, number of participants in each treatment group. 
1378

Subgroup and study, year (Reference)

H. V. T. Ho et al.

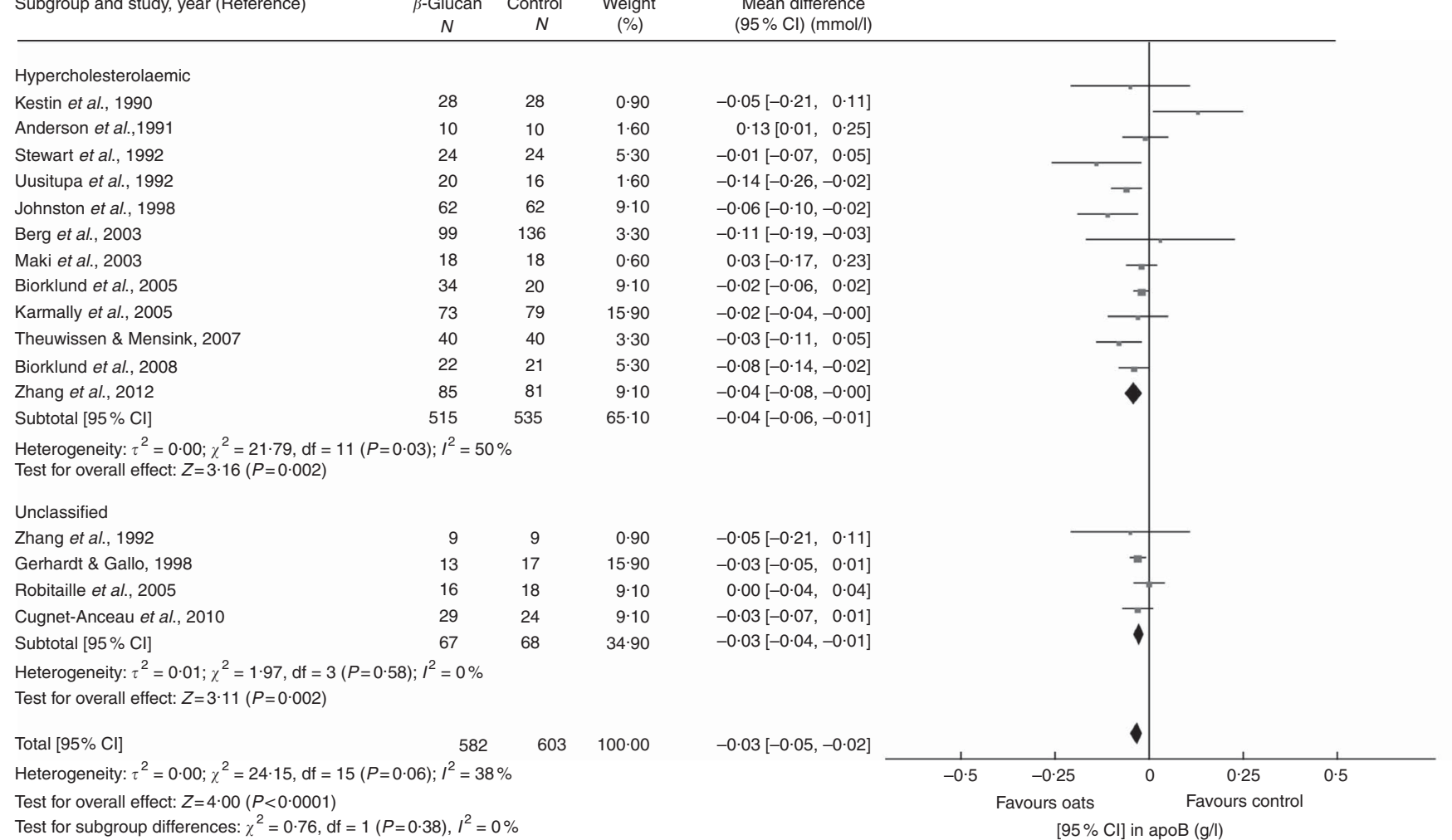

Fig. 4. Forest plot of randomised-controlled trials investigating the effect of oat $\beta$-glucan on apoB. Pooled effect estimate ( $\longrightarrow$ ) for apoB (g/l). Values are mean differences (MD) with $95 \% \mathrm{Cl}$, using the generic inverse-variance random effects models. Inter-study heterogeneity was quantified by $I^{2}$ at a significance of $P<0 \cdot 10$. $N$, Number of participants in each treatment group.

(a) LDL-Cholesterol Begg's test $P=0.061$, Egger's test $P=0.381$

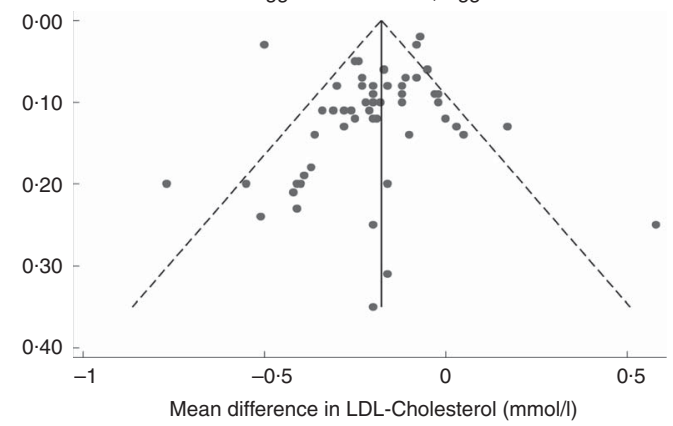

(b) Non-HDL-Cholesterol Begg's test $P=0.528$, Egger's test $P=0.910$

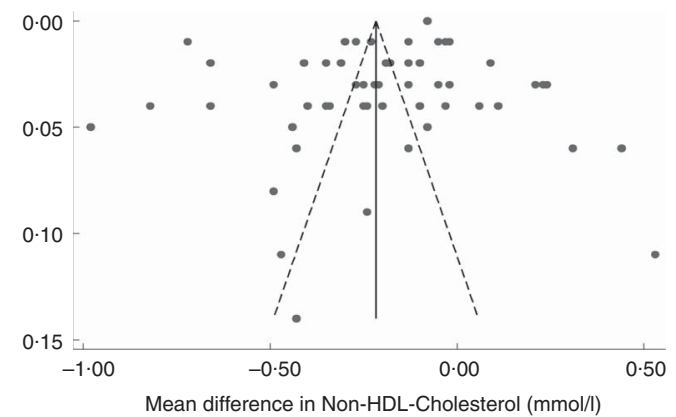

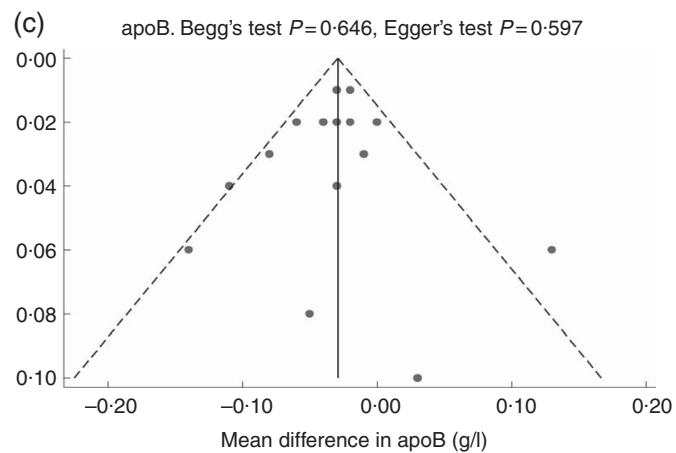

Fig. 5. Publication bias funnel plots. Funnel plots assessing publication bias and effect of small and/or imprecise study effects for (a) LDL-cholesterol, (b) non-HDL-cholesterol and (c) apoB. —_ the pooled effect estimate expressed as the mean difference for each analysis; - - - - - , pseudo-95\% Cl. P-values are derived from quantitative assessment of publication bias by Egger's and Begg's tests. 
LDL-cholesterol levels. In total, twenty-five studies investigating the cholesterol-lowering effect of oats were included in a subgroup analysis, and the authors reported a significant overall LDL-cholesterol reduction of $-0.037 \mathrm{mmol} / 1$ (95\% CI-0.047, $-0.017)$ per $g$ of oat fibre. This is approximately equivalent to $-0.13 \mathrm{mmol} / 1$ per $3.5 \mathrm{~g}, 30 \%$ less than what was observed in our current study (Fig. 2). However, as the results from this meta-analysis were reported as $\mathrm{mmol} / \mathrm{l}$ of LDL-cholesterol reduction per gram of soluble fibre, they cannot be directly compared with the results of the current study.

In the most recent meta-analysis of oat $\beta$-glucan and LDL-cholesterol $^{(18)}$, the authors included twenty-eight RCT and reported an LDL-cholesterol reduction of $-0.25 \mathrm{mmol} / 1(-6 \%)$, whereas this study demonstrated a reduction of $-0.19 \mathrm{mmol} / \mathrm{l}$ $(-4.2 \%)$. This discrepancy could be due to differences in study selection criteria. Whitehead et al. only included RCT that administered $\geq 3 \mathrm{~g} / \mathrm{d}$ of oat $\beta$-glucan, which resulted in a median daily dose of $5 \cdot 1 \mathrm{~g}$, whereas the current meta-analysis included studies of all doses and observed a median dose of $3.5 \mathrm{~g} / \mathrm{d}$. When the results were examined on a per gram basis, LDL-cholesterol reductions were on par (Whitehead et al: $-0.050 \mathrm{mmol} / \mathrm{l} v$., our study: $-0.054 \mathrm{mmol} / \mathrm{l}$ per $\mathrm{g}$ of oat $\beta$-glucan) despite the differences in dose. Interestingly, our meta-regression analysis indicated a significant inverse association between dose and LDL-cholesterol levels (online Supplementary Table S4). Furthermore, when dose was categorised according to Health Canada and US FDA recommendations $(<3.0 v . \geq 3.0 \mathrm{~g} / \mathrm{d})$, there was a trend towards treatment modification by dose $(P=0 \cdot 051)$, such that LDL-cholesterol reduction was almost double in trials that administered $\geq 3.0 \mathrm{~g} / \mathrm{d}$ of oat $\beta$-glucan compared with those that administered $<3.0 \mathrm{~g} / \mathrm{d}$ (online Supplementary Fig. S2). These results further support the health claims set by Health Canada and US FDA that cholesterol lowering can be achieved with a minimum of $3 \mathrm{~g} / \mathrm{d}$ of oat $\beta$-glucan.

This is the first meta-analysis of RCT yielding information on the effect of oat $\beta$-glucan on non-HDL-cholesterol and apoB. These markers have been added to clinical practice guidelines ${ }^{(8,9)}$ on the basis that they are more highly associated with CVD risk than LDL-cholesterol ${ }^{(7)}$. Furthermore, the appreciation of these markers for CVD risk is especially important in adults with the metabolic syndrome and/or diabetes as LDLcholesterol is not typically elevated in this population. Pooled analyses demonstrated significant reductions of non-HDLcholesterol $(-0.20 \mathrm{mmol} / \mathrm{l}(95 \% \mathrm{CI}-0.26,-0 \cdot 15))$ and apoB $(-0.03 \mathrm{~g} / 1(95 \% \mathrm{CI}-0.05,-0.02))$; however, the results are compromised by considerable unexplained heterogeneity. Interestingly, when trials were classified into the hypercholesterolaemic or unclassified group, of which more than a quarter of the studies were conducted in type 2 diabetes mellitus, both categories demonstrated significant reductions in non-HDLcholesterol and apoB. This is an important finding, considering that type 2 diabetes mellitus is generally not associated with increased LDL-cholesterol. Therefore, focusing on interventions that reduce non-HDL-cholesterol and apoB may be more practical and reliable for addressing the increased risk of CVD in type 2 diabetes mellitus.

Effect modification by baseline cholesterol levels has been previously described, such that cholesterol lowering by $\beta$-glucan is generally greater in those with hypercholesterolaemia $^{(9)}$. This was confirmed by our meta-regression analysis demonstrating a significant inverse association between baseline LDL-cholesterol levels and the extent of LDL-cholesterol reduction (online Supplementary Table S4). However, higher baseline levels of non-HDL-cholesterol or apoB were not significantly associated with greater reductions.

There are several limitations to the present meta-analysis that complicate the interpretation of the results. The first one being that the $\beta$-glucan content of oats was estimated for the majority of trials as it was not routinely analysed and reported. As $\beta$-glucan content varies significantly depending on genetics and environmental growing conditions ${ }^{(80,81)}$, it is difficult to precisely measure the treatment effect when the majority of trials did not conduct a chemical analysis of the $\beta$-glucan content of their study products.

Second, the considerable heterogeneity that was observed in LDL-cholesterol and non-HDL-cholesterol was not explained by any of the a priori subgroup analyses. Nevertheless, considering the large number of studies included in this metaanalysis, high heterogeneity is inevitable. The studies included a wide range of food matrices that were used to administer oat $\beta$-glucan, several different processing and storage methods, varying molecular levels of $\beta$-glucan, etc., all of which are interrelated and significantly impact viscosity of the $\beta$-glucan, and thus its cholesterol-lowering potency. Furthermore, nutrition studies have not yet incorporated non-HDL-cholesterol into their primary analysis, despite the simple calculation. Therefore, in addition to all the previously mentioned sources of heterogeneity, the entire set of non-HDL-cholesterol data was mathematically imputed, which may have contributed to the increased heterogeneity.

Irrespective of the large heterogeneity associated with including studies that were conducted in a wide range of participants, in numerous countries, and used various common food products to administer the oat $\beta$-glucan, the results can be considered largely generalisable and indicative that the cholesterol-lowering benefits can be achieved by supplementing oat $\beta$-glucan into commonly consumed foods.

In conclusion, this systematic review and meta-analysis supports the dose-dependent intake of oat $\beta$-glucan for the reduction of LDL-cholesterol, non-HDL-cholesterol and apoB in middle-aged participants. Because of considerable unexplained heterogeneity, caution should be taken when interpreting the results. There is a need for larger, longer, high-quality RCT on the effect of oat $\beta$-glucan on blood cholesterol levels, especially non-HDL-cholesterol and apoB end points, and in participants with different metabolic phenotypes. Special attention should be paid to $\beta$-glucan molecular weight and content in these trials to allow for a more accurate assessment of the cholesterollowering properties of $\beta$-glucan.

\section{Acknowledgements}

The authors thank Teruko Kishibe of Li Ka Shing's International Healthcare Education Centre at St. Michael Hospital for her help in the development of the search strategy. 
The authors' responsibilities were as follows: J. L. S., E. J., A. L. J. and V. V. designed the study; H. V. T. H. and A. Z. conducted the study; H. V. T. H. and S. B. M. analysed data or performed statistical analysis; H. V. T. H. wrote the paper; H. V. T. H. and V. V. had primary responsibility for the final content; all the authors contributed to the critical revision of the article for important intellectual content and approved the final manuscript.

H. V. T. H., A. Z., S. B. M., E. J., F. A.-Y. have no declared conflicts of interest related to this manuscript. J. L. S. has received research support from the Canadian Institutes of Health Research, Canadian Diabetes Association (CDA), PSI Foundation, Calorie Control Council, American Society of Nutrition (ASN), The Coca-Cola Company (investigator initiated, unrestricted), Dr Pepper Snapple Group (investigator initiated, unrestricted), Pulse Canada, The International Tree Nut Council Nutrition Research \& Education Foundation and the INC International Nut and Dried Fruit Council. He has received reimbursement of travel expenses, speaker fees and/or honoraria from the American Heart Association, American College of Physicians, ASN, National Institute of Diabetes and Digestive and Kidney Diseases, CDA, Canadian Nutrition Society, University of South Carolina, University of Alabama at Birmingham, Oldways Preservation Trust, Nutrition Foundation of Italy, Calorie Control Council, Diabetes and Nutrition Study Group (DNSG) of the European Association for the Study of Diabetes (EASD), International Life Sciences Institute (ILSI) North America, ILSI Brazil, Abbott Laboratories, Pulse Canada, Canadian Sugar Institute, Dr Pepper Snapple Group, The CocaCola Company, Corn Refiners Association, World Sugar Research Organization, Dairy Farmers of Canada, Società Italiana di Nutrizione Umana, III World Congress of Public Health Nutrition, C3 Collaborating for Health, White Wave Foods, Rippe Lifestyle, mdBriefcase. He has ad boc consulting arrangements with Winston \& Strawn LLP, Perkins Coie LLP and Tate \& Lyle. He is on the Clinical Practice Guidelines Expert Committee for Nutrition Therapy of both the CDA EASD, and CCS, as well as being on an ASN writing panel for a scientific statement on sugars. He is a member of the International Carbohydrate Quality Consortium and Board Member of the DNSG of the EASD. He serves as an unpaid scientific advisor for the Food, Nutrition, and Safety Program and the Technical Committee on Carbohydrates of the ILSI North America. His wife is an employee of Unilever Canada. V. V. holds a research grant from CDA for study of dietary intervention including viscous, soluble fibre and holds the Canadian $(2,410,556)$ and American $(7,326.404)$ patent on medical use of viscous fibre blend for reducing blood glucose for treatment of diabetes, increasing insulin sensitivity, reduction of systolic blood pressure and blood lipids. A. L. J. is director of research and part owner of Glycemic Index Laboratories, a clinical research organisation.

\section{Supplementary material}

For supplementary material/s referred to in this article, please visit http://dx.doi.org/10.1017/S000711451600341X

\section{References}

1. de Groot A, Luyken R \& Pikaar NA (1963) Cholesterollowering effect of rolled oats. Lancet (London, England) 2, 303-304.

2. Food Directorate Health Products and Food Branch, Health Canada (2010) Oat Products and Blood Cholesterol Lowering. Ottawa: Bureau of Nutritional Sciences.

3. Food and Drug Administration (1997) Food Labeling: Health Claims; Oats and Coronary Heart Disease. Maryland: Health and Human Services.

4. EFSA Panel on Dietetic Products, Nutrition and Allergies (NDA) (2011) Scientific opinion on the substantiation of health claims related to beta-glucans from oats and barley and maintenance of normal blood LDL-cholesterol concentrations (ID 1236, 1299), increase in satiety leading to a reduction in energy intake (ID 851, 852), reduction of post-prandial glycaemic responses (ID 821, 824), and 'digestive function' (ID 850) pursuant to Article 13(1) of Regulation (EC) No 1924/ 2006. EFSA J 9, 2207.

5. The Board of Food Standards Australia New Zealand (2013) Food Standards, Vol. 1.2.7: Nutrition, Health and Related Claims. Canberra: Commonwealth of Australia.

6. Ministry of Health Malaysia (2014) Malaysian dietary guidelines - Key Message 14 - make effective use of nutrition information on food labels. Putrajaya, Malaysia: Ministry of Health Malaysia. www.moh.gov.my/images/gallery/ Garispanduan/diet/km14.pdf (accessed November 2014).

7. Saenger A (2011) Cardiovascular Risk Assessment Beyond LDL Cholesterol: Non-HDL Cholesterol, LDL Particle Number, and Apolipoprotein B. Mayo Clinic Communique. http:// www.mayomedicallaboratories.com/articles/communique/2011/ 11.html (accessed April 2015).

8. National Cholesterol Education Program (NCEP) Expert Panel (2002) Third report on detection, evaluation, and treatment of high blood cholesterol in adults (Adult Treatment Panel III) final report. Circulation 106, 3143-3421.

9. Anderson TJ, Gregoire J, Hegele RA, et al. (2013) 2012 update of the Canadian Cardiovascular Society guidelines for the diagnosis and treatment of dyslipidemia for the prevention of cardiovascular disease in the adult. Can J Cardiol 29, 151-167.

10. The Cochrane Collaboration (2011) Cochrane Handbook for Systematic Reviews of Interventions Version 5.1.0 [J Higgins and S Green, editors]. www.cochrane-handbook.org

11. Moher D, Liberati A, Tetzlaff J, et al. (2009) Preferred reporting items for systematic reviews and meta-analyses: the PRISMA statement. PLoS Med 6, e1000097.

12. Panahi S (2006) The effect of oat beta-glucan on glycemia and blood lipid risk factors for cardiovascular disease. Thesis, University of Toronto.

13. Food and Drug Administration (2009) Labeling \& Nutrition: Guidance for Industry: Evidence-Based Review System for the Scientific Evaluation of Health Claims - Final. http://www.fda. gov/Food/GuidanceRegulation/GuidanceDocumentsRegulatory Information/LabelingNutrition/ucm073332.htm.

14. Greer N, Mosser G, Logan G, et al. (2000) A practical approach to evidence grading. It Comm J Qual Improv 26, 700-712.

15. Kris-Etherton PM \& Dietschy J (1997) Design criteria for studies examining individual fatty acid effects on cardiovascular disease risk factors: human and animal studies. Am J Clin Nutr 65, Suppl., 1590S-1596S.

16. Chen WJ \& Anderson JW (1981) Soluble and insoluble plant fiber in selected cereals and vegetables. Am J Clin Nutr 34, 1077-1082.

17. Anderson JW \& Bridges SR (1988) Dietary fiber content of selected foods. Am J Clin Nutr 47, 440-447. 
18. Whitehead A, Beck EJ, Tosh S, et al. (2014) Cholesterollowering effects of oat beta-glucan: a meta-analysis of randomized controlled trials. Am J Clin Nutr 100, 1413-1421.

19. Heyland DK, Novak F, Drover JW, et al. (2001) Should immunonutrition become routine in critically ill patients? A systematic review of the evidence. JAMA 286, 944-953.

20. Ha V (2013) Effects of dietary pulses on lipid risk factors of cardiovascular disease and oxidative stress. University of Toronto.

21. Elbourne DR, Altman DG, Higgins JP, et al. (2002) Metaanalyses involving cross-over trials: methodological issues. Int J Epidemiol 31, 140-149.

22. Amundsen ÅL, Haugum B \& Andersson H (2003) Changes in serum cholesterol and sterol metabolites after intake of products enriched with an oat bran concentrate within a controlled diet. Scand J Nutr 47, 68-74.

23. Anderson JW, Gilinsky NH, Deakins DA, et al. (1991) Lipid responses of hypercholesterolemic men to oat-bran and wheat-bran intake. Am J Clin Nutr 54, 678-683.

24. Berg A, Konig D, Deibert P, et al. (2003) Effect of an oat bran enriched diet on the atherogenic lipid profile in patients with an increased coronary heart disease risk. A controlled randomized lifestyle intervention study. Ann Nutr Metab 47, 306-311.

25. Biorklund M, van Rees A, Mensink RP, et al. (2011) Changes in serum lipids and postprandial glucose and insulin concentrations after consumption of beverages with beta-glucans from oats or barley: a randomised dose-controlled trial. Eur J Clin Nutr 59, 1272-1281.

26. Biorklund M, Holm J \& Onning G (2008) Serum lipids and postprandial glucose and insulin levels in hyperlipidemic subjects after consumption of an oat beta-glucan-containing ready meal. Ann Nutr Metab 52, 83-90.

27. Braaten JT, Wood PJ, Scott FW, et al. (1994) Oat beta-glucan reduces blood cholesterol concentration in hypercholesterolemic subjects. Eur J Clin Nutr 48, 465-474

28. Bremer JM, Scott RS \& Lintott CJ (1991) Oat bran and cholesterol reduction: evidence against specific effect. Aust $N$ Z J Med 21, 422-426.

29. Charlton KE, Tapsell LC, Batterham MJ, et al. (2012) Effect of 6 weeks' consumption of beta-glucan-rich oat products on cholesterol levels in mildly hypercholesterolaemic overweight adults. Br J Nutr 107, 1037-1047.

30. Davidson MH, Dugan LD, Burns JH, et al. (1991) The hypocholesterolemic effects of beta-glucan in oatmeal and oat bran. A dose-controlled study. JAMA 265, 1833-1839.

31. Demark-Wahnefried W, Bowering J \& Cohen PS (1990) Reduced serum cholesterol with dietary change using fatmodified and oat bran supplemented diets. J Am Diet Assoc 90, 223-229.

32. Johnston L, Reynolds HR, Hunninghake DB, et al. (1998) Cholesterol lower benefits of a whole grain oat readyto-eat cereal. Nutr Clin Care 1, 6-12.

33. Karmally W, Montez MG, Palmas W, et al. (2005) Cholesterollowering benefits of oat-containing cereal in Hispanic Americans. J Am Diet Assoc 105, 967-970.

34. Kerckhoffs DA, Hornstra G \& Mensink RP (2003) Cholesterollowering effect of beta-glucan from oat bran in mildly hypercholesterolemic subjects may decrease when betaglucan is incorporated into bread and cookies. Am J Clin Nutr 78, 221-227.

35. Kestin M, Moss R, Clifton PM, et al. (1990) Comparative effects of three cereal brans on plasma lipids, blood pressure, and glucose metabolism in mildly hypercholesterolemic men. Am J Clin Nutr 52, 661-666.
36. Leadbetter J, Ball MJ \& Mann JI (1991) Effects of increasing quantities of oat bran in hypercholesterolemic people. Am J Clin Nutr 54, 841-845.

37. Lepre F \& Crane S (1992) Effect of oatbran on mild hyperlipidaemia. Med J Aust 157, 305-308.

38. Liatis S, Tsapogas P, Chala E, et al. (2009) The consumption of bread enriched with betaglucan reduces LDL-cholesterol and improves insulin resistance in patients with type 2 diabetes. Diabetes Metab 35, 115-120.

39. Lovegrove JA, Clohessy A, Milon H, et al. (2000) Modest doses of beta-glucan do not reduce concentrations of potentially atherogenic lipoproteins. Am J Clin Nutr 72, 49-55.

40. Maki CK, Davidson HM, Ingram AK, et al. (2003) Lipid responses to consumption of a beta-glucan containing readyto-eat cereal in children and adolescents with mildto-moderate primary hypercholesterolemia. Nutr Res $\mathbf{2 3}$, $1527-1535$

41. Maki KC, Beiseigel JM, Jonnalagadda SS, et al. (2010) Wholegrain ready-to-eat oat cereal, as part of a dietary program for weight loss, reduces low-density lipoprotein cholesterol in adults with overweight and obesity more than a dietary program including low-fiber control foods. J Am Diet Assoc 110, 205-214.

42. Mårtensson $\mathrm{O}$, Biörklund $\mathrm{M}$, Lambo $\mathrm{AM}$, et al. (2005) Fermented, ropy, oat-based products reduce cholesterol levels and stimulate the bifidobacteria flora in humans. Nutr Res $\mathbf{2 5}$, 429-442.

43. Momenizadeh A, Heidari R, Sadeghi M, et al. (2014) Effects of oat and wheat bread consumption on lipid profile, blood sugar, and endothelial function in hypercholesterolemic patients: a randomized controlled clinical trial. ARYA Atheroscler 10, 259-265.

44. Noakes M, Clifton PM, Nestel PJ, et al. (1996) Effect of highamylose starch and oat bran on metabolic variables and bowel function in subjects with hypertriglyceridemia. Am J Clin Nutr 64, 944-951.

45. Onning G, Wallmark A, Persson M, et al. (1999) Consumption of oat milk for 5 weeks lowers serum cholesterol and LDL cholesterol in free-living men with moderate hypercholesterolemia. Ann Nutr Metab 43, 301-309.

46. Queenan KM, Stewart ML, Smith KN, et al. (2007) Concentrated oat beta-glucan, a fermentable fiber, lowers serum cholesterol in hypercholesterolemic adults in a randomized controlled trial. Nutr $J \mathbf{6}, 6$.

47. Reyna-Villasmil N, Bermudez-Pirela V, Mengual-Moreno E, et al. (2007) Oat-derived beta-glucan significantly improves HDLC and diminishes LDLC and non-HDL cholesterol in overweight individuals with mild hypercholesterolemia. Am J Ther 14, 203-212.

48. Reynolds HR, Quiter E \& Hunninghake DB (2000) Whole grain oat cereal lowers serum lipids. Top Clin Nutr 15, 74-83.

49. Romero AL, Romero JE, Galaviz S, et al. (1998) Cookies enriched with psyllium or oat bran lower plasma LDL cholesterol in normal and hypercholesterolemic men from Northern Mexico. J Am Coll Nutr 17, 601-608.

50. Stewart FM, Neutze JM \& Newsome-White R (1992) The addition of oatbran to a low fat diet has no effect on lipid values in hypercholesterolaemic subjects. NZ Med J 105, 398-400.

51. Theuwissen E \& Mensink RP (2007) Simultaneous intake of beta-glucan and plant stanol esters affects lipid metabolism in slightly hypercholesterolemic subjects. J Nutr 137, 583-588.

52. Thongoun P, Pavadhgul P, Bumrungpert A, et al. (2013) Effect of oat consumption on lipid profiles in hypercholesterolemic adults. J Med Assoc Thai 96, Suppl. 5, S25-S32. 
53. Turnbull WH \& Leeds AR (1987) Reduction of total and LDL-cholesterol in plasma by rolled oats. J Clin Nutr Gastroentrol 2, 177-181.

54. Uusitupa MI, Ruuskanen E, Makinen E, et al. (1992) A controlled study on the effect of beta-glucan-rich oat bran on serum lipids in hypercholesterolemic subjects: relation to apolipoprotein E phenotype. J Am Coll Nutr 11, 651-659.

55. Van Horn L, Moag-Stahlberg A, Liu KA, et al. (1991) Effects on serum lipids of adding instant oats to usual American diets. Am J Public Health 81, 183-188.

56. Van Horn L, Liu K, Gerber J, et al. (2001) Oats and soy in lipidlowering diets for women with hypercholesterolemia: is there synergy? J Am Diet Assoc 101, 1319-1325.

57. Whyte JL, McArthur R, Topping D, et al. (1992) Oat bran lowers plasma cholesterol levels in mildly hypercholesterolemic men. J Am Diet Assoc 92, 446-449.

58. Wolever TM, Tosh SM, Gibbs AL, et al. (2010) Physicochemical properties of oat $\beta$-glucan influence its ability to reduce serum LDL cholesterol in humans: a randomized clinical trial. Am J Clin Nutr 92, 723-732.

59. Zhang J, Li L, Song P, et al. (2012) Randomized controlled trial of oatmeal consumption versus noodle consumption on blood lipids of urban Chinese adults with hypercholesterolemia. Nutr J 11, 54 .

60. Beck EJ, Tapsell LC, Batterham MJ, et al. (2010) Oat betaglucan supplementation does not enhance the effectiveness of an energy-restricted diet in overweight women. BrJ Nutr $\mathbf{1 0 3}$, $1212-1222$.

61. Chen J, He J, Wildman RP, et al. (2006) A randomized controlled trial of dietary fiber intake on serum lipids. Eur J Clin Nutr 60, 62-68.

62. Cugnet-Anceau C, Nazare JA, Biorklund M, et al. (2010) A controlled study of consumption of beta-glucan-enriched soups for 2 months by type 2 diabetic free-living subjects. $\mathrm{BrJ}$ Nutr 103, 422-428.

63. Davy BM, Davy KP, Ho RC, et al. (2002) High-fiber oat cereal compared with wheat cereal consumption favorably alters LDL-cholesterol subclass and particle numbers in middle-aged and older men. Am J Clin Nutr 76, 351-358.

64. Gerhardt AL \& Gallo NB (1998) Full-fat rice bran and oat bran similarly reduce hypercholesterolemia in humans. J Nutr $\mathbf{1 2 8}$, 865-869.

65. Gold KV \& Davidson DM (1988) Oat bran as a cholesterolreducing dietary adjunct in a young, healthy population. West J Med 148, 299-302.

66. Ibrugger S, Kristensen M, Poulsen MW, et al. (2013) Extracted oat and barley beta-glucans do not affect cholesterol metabolism in young healthy adults. $J$ Nutr $\mathbf{1 4 3}$ $1579-1585$.
67. Kabir M, Oppert JM, Vidal H, et al. (2002) Four-week lowglycemic index breakfast with a modest amount of soluble fibers in type 2 diabetic men. Metabolism 51, 819-826.

68. Ma X, Gu J, Zhang Z, et al. (2013) Effects of Avena nuda L. on metabolic control and cardiovascular disease risk among Chinese patients with diabetes and meeting metabolic syndrome criteria: secondary analysis of a randomized clinical trial. Eur J Clin Nutr 67, 1291-1297.

69. McGeoch SC, Johnstone AM, Lobley GE, et al. (2013) A randomized crossover study to assess the effect of an oat-rich diet on glycaemic control, plasma lipids and postprandial glycaemia, inflammation and oxidative stress in type 2 diabetes. Diabet Med 30, 1314-1323.

70. Naumann E, van Rees AB, Onning G, et al. (2006) Beta-glucan incorporated into a fruit drink effectively lowers serum LDL-cholesterol concentrations. Am J Clin Nutr 83, 601-605.

71. Pick ME, Hawrysh ZJ, Gee MI, et al. (1996) Oat bran concentrate bread products improve long-term control of diabetes: a pilot study. I Am Diet Assoc 96, 1254-1261.

72. Pins JJ, Geleva D, Keenan JM, et al. (2002) Do whole-grain oat cereals reduce the need for antihypertensive medications and improve blood pressure control? J Fam Pract 51, 353-359.

73. Poulter N, Chang CL, Cuff A, et al. (1994) Lipid profiles after the daily consumption of an oat-based cereal: a controlled crossover trial. Am J Clin Nutr 59, 66-69.

74. Robitaille J, Fontaine-Bisson B, Couture P, et al. (2005) Effect of an oat bran-rich supplement on the metabolic profile of overweight premenopausal women. Ann Nutr Metab 49, 141-148.

75. Saltzman E, Das SK, Lichtenstein AH, et al. (2001) An oatcontaining hypocaloric diet reduces systolic blood pressure and improves lipid profile beyond effects of weight loss in men and women. J Nutr 131, 1465-1470.

76. Swain JF, Rouse IL, Curley CB, et al. (1990) Comparison of the effects of oat bran and low-fiber wheat on serum lipoprotein levels and blood pressure. $N$ Eng J Med 322, 147-152.

77. Van Horn L, Emidy LA, Liu KA, et al. (1988) Serum lipid response to a fat-modified, oatmeal-enhanced diet. Prev Med 17, 377-386.

78. Zhang JX, Hallmans G, Andersson H, et al. (1992) Effect of oat bran on plasma cholesterol and bile acid excretion in nine subjects with ileostomies. Am J Clin Nutr 56, 99-105.

79. Brown L, Rosner B, Willett WW, et al. (1999) Cholesterollowering effects of dietary fiber: a meta-analysis. Am J Clin Nutr 69, 30-42.

80. Bartlomiej S, Justyna RK \& Ewa N (2012) Bioactive compounds in cereal grains - occurrence, structure, technological significance and nutritional benefits - a review. Food Sci Technol Int 18, 559-568.

81. Lee CJ, Horsley RD, Manthey FA, et al. (1997) Comparisons of $\beta$-glucan content of barley and oat. Cereal Chem J 74, 571-575. 